\title{
217 DIFFERENTIATION OF PLURIPOTENT HUMAN STEM CELLS TO EPICARDIUM AND EPICARDIUM-DERIVED CORONARY SMOOTH MUSCLE CELLS
}

\footnotetext{
D lyer, ${ }^{1,2}$ S Sinha ${ }^{1,2}{ }^{1}$ The Anne McLaren Laboratory for Regenerative Medicine; ${ }^{2}$ Division of Cardiovascular Medicine, University of Cambridge, Addenbrooke's hospital, Cambridge, UK
}

doi:10.1136/heartjnl-2013-304019.217

Cardiovascular regenerative medicine is an emerging field that intersects both developmental biology and clinical research. Pluripotent human stem cells (hPSCs) offer unique value to this field as they can be directed to become specialised cardiovascular cells. Due to its fundamental role in heart development, the epicardium has emerged as a multipotent cardiovascular progenitor source with therapeutic potential in terms of coronary smooth muscle cell (CoSMC), cardiac fibroblast and cardiomyocyte regeneration. We recently reported an in vitro strategy for generating lineage-specific vascular smooth muscle cells (VSMCs) from hPSCs (Cheung et al. Nature Biotechnol 2012;30:165-73). We have now extended this approach to obtain epicardium and its derivatives. Here we investigated the function of key developmental growth factors such as Wnt3a, BMP4, FGF-2 and retinoic acid (RA) in guiding hPSCs to an epicardial lineage through lateral plate mesoderm under chemically defined conditions. Our results reveal that a combination of Wnt3a, BMP4 and RA induces high expression of epicardium specific genes (Tbx18, Tcf21, Wt1 and Basonuclin) comparable to human fetal epicardial explants. The hPSC-derived epicardial cells, when treated with PDGF-BB, TGF- $\beta 1$ and Thymosin- $\beta 4$, resulted in differentiated VSMCs that expressed a 
repertoire of VSMC markers. Ongoing studies will validate whether the epicardium-derived VSMCs are coronary artery-like and identify factors that promote generation of other epicardium derived cells such as cardiac fibroblasts and cardiomyocytes. We anticipate that coupling epicardial cells with receptor specific tissue-engineered scaffolds may direct them selectively to a CoSMC or cardiomyocyte lineage and lay the foundation for novel approaches to regenerative cardiovascular medicine. 